
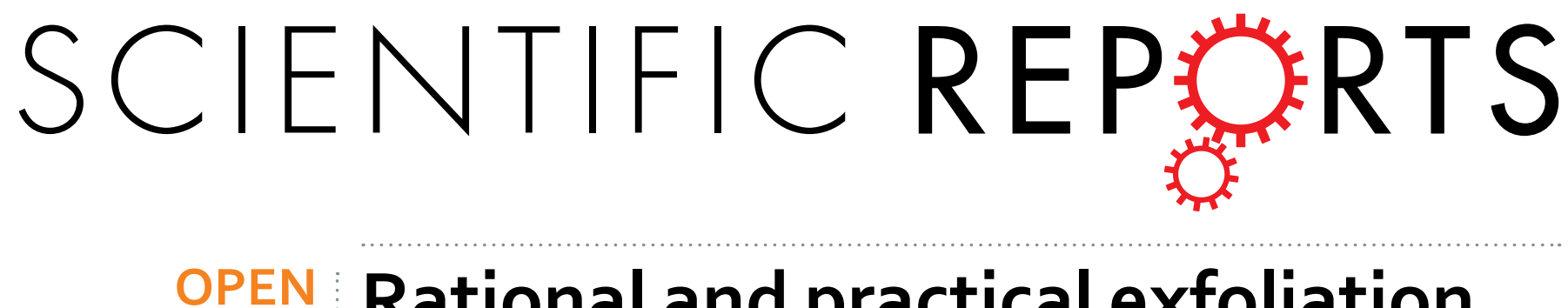

\title{
Rational and practical exfoliation of graphite using well-defined poly(3-hexylthiophene) for the
}

Received: 20 September 2016

Accepted: 29 November 2016

Published: 06 January 2017

\section{preparation of conductive polymer/ graphene composite}

Hiroki Iguchi ${ }^{1}$, Chisato Higashi ${ }^{1}$, Yuichi Funasaki ${ }^{1}$, Keisuke Fujita ${ }^{1}$, Atsunori Mori ${ }^{1}$, Akira Nakasuga' ${ }^{2}$ Tatsuo Maruyama ${ }^{1}$

Processing and manipulation of highly conductive pristine graphene in large quantities are still major challenges in the practical application of graphene for electric device. In the present study, we report the liquid-phase exfoliation of graphite in toluene using well-defined poly(3-hexylthiophene) (P3HT) to produce a $\mathrm{P} 3 \mathrm{HT} / \mathrm{graphene}$ composite. We synthesize and use regioregular $\mathrm{P} 3 \mathrm{HT}$ with controlled molecular weights as conductive dispersants for graphene. Simple ultrasonication of graphite flakes with the $\mathrm{P} 3 \mathrm{HT}$ successfully produces single-layer and few-layer graphene sheets dispersed in toluene. The produced $\mathrm{P} 3 \mathrm{HT} /$ graphene composite can be used as conductive graphene ink, indicating that the $\mathrm{P} 3 \mathrm{HT} /$ graphene composite has high electrical conductivity owing to the high conductivity of $\mathrm{P} 3 \mathrm{HT}$ and graphene. The $\mathrm{P} 3 \mathrm{HT} / \mathrm{graphene}$ composite also works as an oxidation-resistant and conductive film for a copper substrate, which is due to the high gas-barrier property of graphene.

Graphene is one of the most interesting nanomaterials owing to its unique properties, such as high electrical conductivity, high mechanical toughness, high gas-barrier performance, and unique optical properties ${ }^{1-3}$. Industrial applications of graphene are expected in a wide range of areas, including electronic materials and medical engineering. In particular, its application in electronics has been highly expected and widely studied. Since the pioneering work on mechanical exfoliation for the production of graphene in $2004^{1}$, a number of methods for the production of graphene have been proposed: for example, mechanical exfoliation ${ }^{1}$, chemical vapor deposition (CVD) ${ }^{4,5}$, heat decomposition ${ }^{6}$, and chemical synthesis 7 . Many of these methods are costly, ineffective, and non-scalable. 'Liquid-phase exfoliation' of graphite is one of the most inexpensively scalable approaches. However, graphene usually aggregates in solvents because of the strong hydrophobic interaction and van der Waals force between graphene sheets. The aggregation causes a loss of favorable properties and increases the difficulty for the practical manipulation of graphene. Liquid-phase exfoliation is divided into chemical and physical processes. Ever since chemically exfoliated graphene (e.g., graphene oxide, acidified graphene, and reduced graphene oxide) was shown to have a number of structural defects ${ }^{8,9}$, recent studies have focused on a physical process, especially on pristine graphene produced by the liquid-phase exfoliation of graphite. Various types of dispersants (surfactants, polymers, ionic liquids, proteins, and nanotubes) have been proposed for the direct production of pristine graphene from graphit ${ }^{10-16}$. Many of these dispersants, however, decrease the electrical conductivity of graphene because they disrupt the extended aromatic conjugated systems and cover the graphene with nonconductive molecules ${ }^{16,17}$.

Poly(3-hexylthiophene) (P3HT) has attracted attention in fields such as material science and electronics owing to its high electrical conductivity and its processability ${ }^{18}$. Although $\pi-\pi$ interactions between P3HT and carbon nanomaterials (carbon nanotubes and graphene oxides) have been observed ${ }^{19-24}$, there is, to the best

${ }^{1}$ Department of Chemical Science and Engineering, Graduate School of Engineering, Kobe University, 1-1 Rokkodaicho, Nada-ku, Kobe 657-8501, Japan. ${ }^{2}$ Sekisui Chemical Co., Ltd., 2-1 Hyakuyama, Shimamoto-cho, Mishima-gun, Osaka 618-0021, Japan. Correspondence and requests for materials should be addressed to T.M. (email: tmarutcm@crystal.kobe-u.ac.jp) 


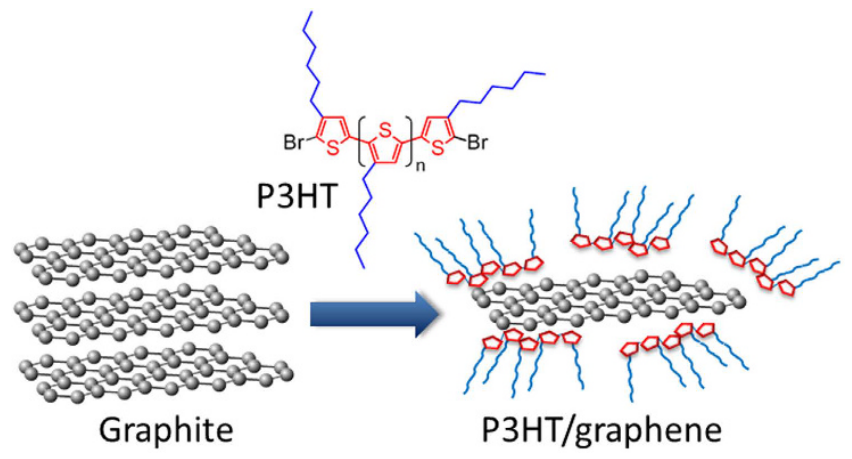

Figure 1. Schematic illustration of the exfoliation of graphite and the graphene dispersion using poly(3hexylthiophene).

of our knowledge, no report on the exfoliation of pristine graphite using P3HT to produce a stable dispersion of graphene. Regioregularity of $\mathrm{P} 3 \mathrm{HT}$ plays an important role in the electrical conductivity of $\mathrm{P} 3 \mathrm{HT}^{18}$. Our group has already succeeded in the regiochemically-controlled synthesis of P3HT having controlled molecular weights $^{25-27}$. In this study, we propose the exfoliation of pristine graphite in toluene using well-defined P3HT as a conductive dispersant to produce pristine graphene without loss of the electrical conductivity (Fig. 1). The present study also demonstrates that the $\mathrm{P} 3 \mathrm{HT} /$ graphene composite can be used as conductive ink and an oxidation-resistant film for a metal substrate.

\section{Methods}

Materials. Graphite flakes and copper-plated divinylbenzene microparticles (CuMPs) were provided by Sekisui Chemical Co., Ltd. (Tokyo, Japan). 2-Bromo-3-hexylthiophene and [1,3-bis(2,6-diisopropylphenyl)imidazol-2-ylidene] triphenylphosphine nickel(II) dichloride $\left(\mathrm{NiCl}_{2}\left(\mathrm{PPh}_{3}\right) \mathrm{IPr}\right)$ were purchased from Tokyo Chemical Industries, Ltd. (Tokyo, Japan). 2,2,6,6-Tetramethylpiperidinylmagnesium chloride lithium chloride complex (TMPMgCl-LiCl) solution was purchased from Sigma (St. Louis, MO). Other chemicals were purchased from Wako PureChemical Industries, Ltd. (Osaka, Japan). The water used was high-quality deionized water (Milli-Q water, $>15 \mathrm{M} \Omega \mathrm{cm}^{-1}$ ) produced using a Milli-Q Advantage A100 system equipped with an Elix UV 3 system (Millipore, Molsheim, France).

Polymerization of 2-bromo-3-hexylthiophene using a Knochel-Hauser base. P3HT was synthesized by the slightly modified procedure previously reported by $\mathrm{us}^{25-27}$. To a THF solution containing $1.0 \mathrm{M}$ TMPMgCl-LiCl (1.56 mmol), 2-bromo-3-hexylthiophene $(1.3 \mathrm{mmol}, 324 \mathrm{mg})$ was added dropwise at $25^{\circ} \mathrm{C}$. After stirring at $25^{\circ} \mathrm{C}$ for $3 \mathrm{~h}$, THF $(20 \mathrm{~mL})$ and $\mathrm{NiCl}_{2}\left(\mathrm{PPh}_{3}\right) \operatorname{IPr}(28.3 \mathrm{mg}, 0.036 \mathrm{mmol})$ were successively added. The resulting mixture was stirred at $25^{\circ} \mathrm{C}$ for $1 \mathrm{~h}$. Hydrochloric acid $(1.0 \mathrm{M}, 20 \mathrm{~mL})$ and methanol $(20 \mathrm{~mL})$ were added to form a precipitate. The mixture was filtered and the residue was washed with methanol and $n$-hexane repeatedly to obtain a dark purple solid, which was dried under reduced pressure to yield $144 \mathrm{mg}$ of poly(3-hexylthiophene) (P3HT) (66\%). Molecular weights and the molecular weight distribution of P3HT were estimated by SEC analysis (eluent: $\mathrm{CHCl}_{3}$ ) using polystyrene as the molecular weight standard. For example, SEC analysis showed $M_{\mathrm{n}}=6000$, and $M_{\mathrm{w}} / M_{\mathrm{n}}=1.10$. The regioregularity was estimated by ${ }^{1} \mathrm{H}$ NMR analysis (thienyl- $\mathrm{CH}_{2}-$ signals) at the $\delta 2.80(\mathrm{H}-\mathrm{T})$ and $\delta 2.60(\mathrm{H}-\mathrm{H})$ signals $(\mathrm{H}-\mathrm{T}$ regioregularity $=97 \%)$.

Regio-irregular P3HT (H-T regioregularity 77\%, Mn 7000 and $M_{w} / M_{n}=1.6$ ) was synthesized according to a previous report ${ }^{28}$.

Preparation of graphene dispersion with P3HT. Graphite flakes were ultrasonicated (AsOne Corporation., Ultrasonic cleaner VS-D100, $31 \mathrm{kHz}$ ) with P3HT in toluene at $80^{\circ} \mathrm{C}$ for $30 \mathrm{~min}$, followed by incubation at $30^{\circ} \mathrm{C}$ for $15 \mathrm{~min}$. The concentration of graphite flakes was $0.33 \mathrm{mg} \mathrm{mL}^{-1}$. By adding the different quantities of $\mathrm{P} 3 \mathrm{HT}$, the mixed ratios of $\mathrm{P} 3 \mathrm{HT} /$ graphite were varied to $0,0.1,0.2,0.5,0.75,1.0$, and $1.5 \mathrm{wt} / \mathrm{wt}$. Following ultrasonication, the dispersions were centrifuged (Hitachi Koki Co., Ltd., CF15RX) at $1000 \times g$ for $20 \mathrm{~min}$ to remove the unexfoliated particles and thick flakes of graphite. Then, the supernatant was collected as the graphene dispersion.

Preparation of $\mathrm{P} 3 \mathrm{HT} / \mathrm{graphene}$ composite film-paper. $\mathrm{P} 3 \mathrm{HT} /$ graphene composite film-papers were prepared by vaccum filtration. Graphene dispersions $(3 \mathrm{ml}$ ) containing $42 \mu \mathrm{g}$ graphene, which were prepared using P3HT $\left(M_{\mathrm{n}}=6,000\right)$ or SDS, were filtrated through filter paper that were $8 \mathrm{~mm}$ in diameter and $4 \mu \mathrm{m}$ of pore size (Kiriyama Grass Co., Ltd.). P3HT/graphene (or SDS/graphene) on the filter paper was dried at $100^{\circ} \mathrm{C}$ for $5 \mathrm{~min}$ to ensure complete solvent evaporation.

Preparation of $\mathrm{P} 3 \mathrm{HT} / \mathrm{graphene-coated} \mathrm{CuMPs.} \mathrm{The} \mathrm{CuMPs} \mathrm{were} \mathrm{rinsed} \mathrm{with} \mathrm{H}_{2} \mathrm{SO}_{4}$ aqueous solution $(0.1 \mathrm{M})$ for $10 \mathrm{~min}$ at $25^{\circ} \mathrm{C}$ to remove copper oxide from the surfaces. Then, they were rinsed with excess amounts of water and ethanol three times each. P3HT- and P3HT/graphene-coated CuMPs were prepared by dip-coating the rinsed CuMPs $(20 \mathrm{mg})$ in $\mathrm{P} 3 \mathrm{HT} /$ graphene dispersion $(400 \mu \mathrm{L})$ for $1.5 \mathrm{~h}$ at $40^{\circ} \mathrm{C}$, followed by the removal of the supernatant by vaccum evaporation overnight to dry the P3HT/graphene-coated CuMPs. 

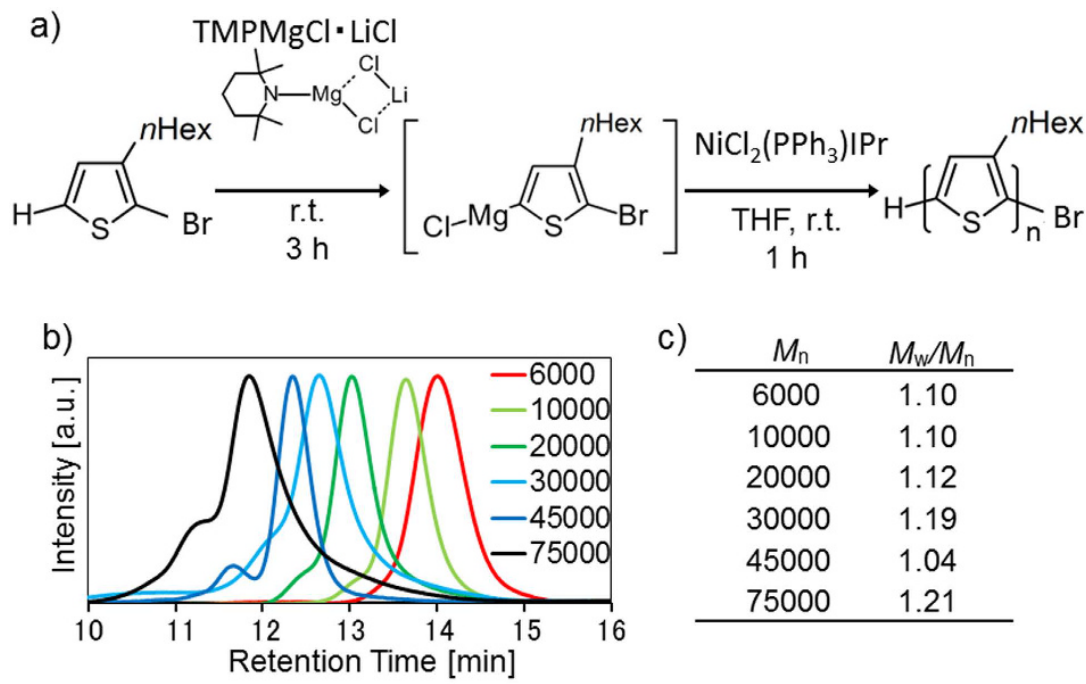

c)

\begin{tabular}{cc}
$M_{\mathrm{n}}$ & $M_{\mathrm{w}} / M_{\mathrm{n}}$ \\
\hline 6000 & 1.10 \\
10000 & 1.10 \\
20000 & 1.12 \\
30000 & 1.19 \\
45000 & 1.04 \\
75000 & 1.21 \\
\hline
\end{tabular}

Figure 2. (a) Polymerization of 2-bromo-3-hexylthiophene using the Knochel-Hauser base and a nickel catalyst. (b) Size-exclusion chromatography (SEC) profiles of synthesized P3HT with different $M_{\mathrm{n}}$. (c) Numberaverage molecular weights and polydispersity indices $\left(M_{\mathrm{w}} / M_{\mathrm{n}}\right)$ of synthesized P3HT.

Sample characterization. UV/Vis absorption spectra of the dispersions (200-900 $\mathrm{nm}$ ) were recorded using a double-beam spectrophotometer (JASCO, V-770). Since P3HT did not exhibit an absorbance around $660 \mathrm{~nm}$, absorption measurements at $660 \mathrm{~nm}$ allowed us to evaluate the concentration of dispersed graphene in toluene.

Raman spectroscopy measurements were performed on a Nano-Raman system WITec alpha300R using a laser excitation at $532 \mathrm{~nm}$ (Nd-YAG-neodymium-doped yttrium aluminium garnet type laser) at $3 \mathrm{~mW}$.

TEM images were obtained using a JEOL $2100 \mathrm{~F}$ transmission electron microscope operated at $200 \mathrm{kV}$. A drop of graphene dispersion was placed on a copper grid covered with elastic carbon films manufactured by Okenshoji Co., Ltd. and dried under vaccum at ambient temperature.

AFM images were obtained in a tapping mode using Hitachi Nanonavi E-sweep with cantilevers at $17,000 \mathrm{~N} \mathrm{~m}^{-1}$. SEM observations were performed using a JEOL JSM-7500F scanning electron microscope.

Electrical resistance measurements were performed using a four-point-probe ohmmeter (Mitsubishi Chemical Analytec Co., Ltd. LorestaAX MCP-T370, TFP-prove) for P3HT/graphene composite paper films and a digital multimeter (Custom Co., Ltd. CDM-200D) for CuMPs.

\section{Results}

Regioregular P3HT with controlled molecular weights were synthesized according to our previous report (Fig. 2a) $)^{25}$. Figure $2 \mathrm{~b}$ shows the elution profiles of the synthesized P3HT from size-exclusion chromatography (SEC). The SEC profiles reveal that the synthesized P3HT had $M_{\mathrm{n}}$ ranging from 6,000 to 75,000 with a very narrow distribution of molecular weights $\left(M_{\mathrm{w}} / M_{\mathrm{n}}\right.$ was from 1.04 to 1.21 , Fig. $\left.2 \mathrm{c}\right)$, which agrees with our previous reports ${ }^{25-27}$.

Graphite flakes (Fig. S1, Supporting Information) were mixed with P3HT in toluene and ultrasonicated at $80^{\circ} \mathrm{C}$ for $30 \mathrm{~min}$. After centrifugation, the resultant toluene solution containing P3HT and graphene was dark brown (inset of Fig. 3a). Figure S2 shows the absorption spectra of the P3HT and P3HT/graphene solutions. The P3HT/graphene solution exhibits a plateau absorbance over $600 \mathrm{~nm}$, which was not observed in the P3HT solution. These results indicate the exfoliation of graphite by $\mathrm{P} 3 \mathrm{HT}$ in toluene to produce the $\mathrm{P} 3 \mathrm{HT} /$ graphene dispersion $^{12}$.

We evaluated the effect of the $M_{\mathrm{n}}$ of $\mathrm{P} 3 \mathrm{HT}$ on the dispersing graphene by measuring the absorbance of the $\mathrm{P} 3 \mathrm{HT} /$ graphene dispersion at $660 \mathrm{~nm}^{12}$. Figure 3a reveals that low-molecular-weight P3HT $\left(M_{\mathrm{n}}=6,000\right)$ was the most effective in dispersing graphene. $\mathrm{P} 3 \mathrm{HT}$ with molecular weights of more than 40,000 resulted in low graphene concentrations in the dispersion, which might be related to the low solubility of the high-molecular-weight P3HT in toluene ${ }^{18,29}$.

The effect of the $\mathrm{P} 3 \mathrm{HT} /$ graphite ratio on dispersing graphene was also investigated (Fig. $3 \mathrm{~b}$ ). As the ratio was increased up to 1.0, the dispersing ability of P3HT increased. At a ratio of 1.5 , the dispersing ability slightly decreased. When P3HT with $M_{\mathrm{n}} 6,000$ was used and the P3HT/graphite was 1.0, the graphene concentration in toluene was $14 \mu \mathrm{g} \mathrm{mL}^{-1}$, in which the yield of graphene was $3.5 \%$.

We also used relatively regio-irregular P3HT (H-T regioregularity $77 \%, M_{n} 7000$ and $M_{\mathrm{w}} / M_{\mathrm{n}}=1.6$ ) at a P3HT/ graphite ratio of 1.0 to exfoliate graphite and to disperse graphene in toluene. Interestingly, there observed negligible absorbance at $660 \mathrm{~nm}$ (Fig. S3), meaning almost no exfoliation of graphite and no graphene dispersion. The twisted structure of the regio-irregular P3HT might inhibit its intercalation into graphite and might be unable to form a stable composite with a graphene sheet. These results proved the importance of the P3HT regioregularity in the exfoliation of graphite and in the dispersion of graphene. The following experiments were carried out using regioregular P3HT with $M_{\mathrm{n}}$ of 6,000 and at a P3HT/graphite ratio of 1.0. 

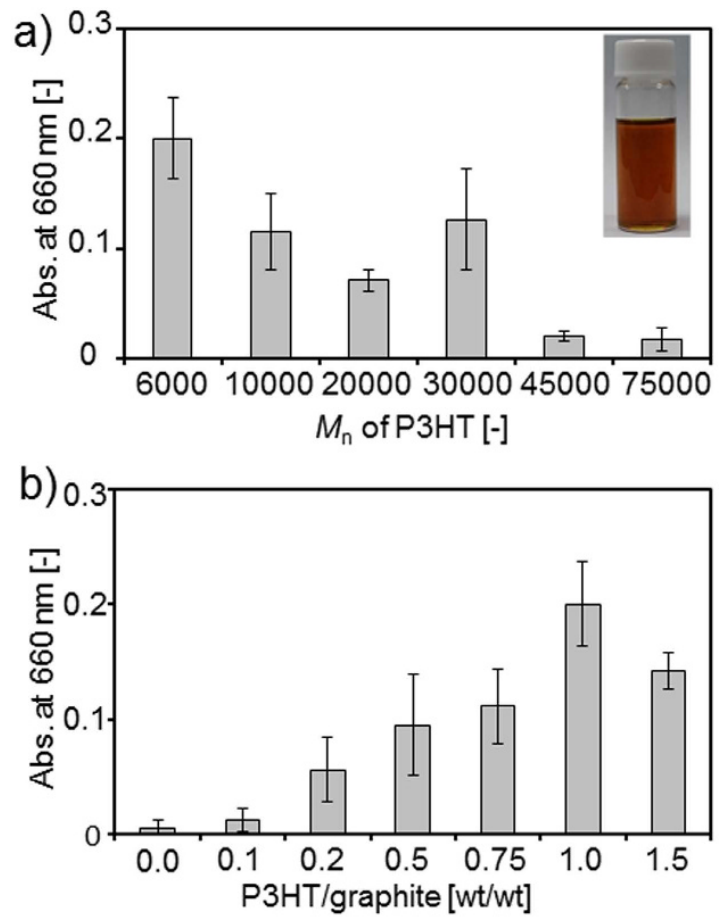

Figure 3. Absorbance at $660 \mathrm{~nm}$ of graphene dispersions in toluene. (a) Effect of different $M_{\mathrm{n}}$ of P3HT (the $\mathrm{P} 3 \mathrm{HT} /$ graphite ratio was 1.0) and (b) effect of different mixed ratios of P3HT $\left(M_{\mathrm{n}}=6,000\right) /$ graphite on the absorbance. The concentration of graphite flakes was $0.33 \mathrm{mg} \mathrm{mL}^{-1}$. The inset represents a photo of a P3HT $\left(M_{\mathrm{n}}=6000\right) /$ graphene dispersion.

The P3HT/graphene dispersion was characterized using a transmission electron microscope (TEM), an atomic force microscope (AFM), and Raman spectroscopy. TEM images of the P3HT/graphene dispersion are shown in Fig. $4 \mathrm{a}$ and b. Large graphene sheets with lateral dimensions of $0.1-2 \mu \mathrm{m}$ were observed. Tri-layer and single-layer graphene were observed by considering the edge of the graphene in Fig. 4a and b, respectively. Nanowires of P3HT were also observed, which is consistent with a previous report ${ }^{21}$. Figure $4 \mathrm{c}$ shows the AFM image of the $\mathrm{P} 3 \mathrm{HT} /$ graphene dispersion deposited on a mica substrate and the height profile along the red line in the figure revealed that the thickness of $\mathrm{P} 3 \mathrm{HT} /$ graphene was $2.6 \mathrm{~nm}$, which corresponded to tri-layer graphene with $\mathrm{P}_{3} \mathrm{HT}^{15}$. These observations clearly demonstrate that graphite flakes were exfoliated by $\mathrm{P} 3 \mathrm{HT}$ to produce single-layer and few-layer graphene sheets.

The above investigations suggest that $\mathrm{P} 3 \mathrm{HT}$ works as a dispersant for graphene in toluene. Defects in graphene often occur during the exfoliation of graphite and dispersion of graphene in solvents ${ }^{30,31}$. We measured the Raman spectra of graphite flakes and the dry P3HT/graphene composite. Figure $4 \mathrm{~d}$ shows the D band $\left(1360 \mathrm{~cm}^{-1}\right), \mathrm{G}$ band $\left(1584 \mathrm{~cm}^{-1}\right)$, and $2 \mathrm{D}$ band $\left(2680 \mathrm{~cm}^{-1}\right)$ of the analytes 32,33 . The D/G band intensity ratio indicates the degree of structural defects in graphene $e^{32}$. The dry P3HT/graphene composite showed an $I_{\mathrm{D}} / I_{\mathrm{G}}$ ratio of 0.63 , which was almost the same as that of the graphite flakes. These measurements indicate that defects in the graphene sheet did not occur during the P3HT-induced exfoliation of the graphite and graphene dispersion.

To investigate the electrical property of the $\mathrm{P} 3 \mathrm{HT}$ /graphene composite, $\mathrm{P} 3 \mathrm{HT} /$ graphene and sodium dodecyl sulfate (SDS)/graphene composites on filter paper were prepared by vacuum filtration of the graphene dispersion (Fig. 5a) ${ }^{34}$. The sheet resistance of the P3HT/graphene composite was $1.8 \pm 0.5 \mathrm{k} \Omega s q^{-1}$, which was much smaller than that of the SDS/graphene composite $\left(31.6 \pm 3.0{\mathrm{k} \Omega \mathrm{sq}^{-1}}^{-1}\right.$. The resistance of the $\mathrm{P} 3 \mathrm{HT} /$ graphene-composite was comparable with those of graphene films with other dispersants ${ }^{11,15,35}$, of CVD-grown graphene ${ }^{36,37}$ and of commercially available graphene ${ }^{38}$. We assembled the P3HT/graphene composite with a circuit and succeeded in energization (LED lighting in Fig. 5b). The remarkably low resistance of the P3HT/graphene composite arose from the high conductivity of $\mathrm{P} 3 \mathrm{HT}$. These investigations proved the great potential of the $\mathrm{P} 3 \mathrm{HT} /$ graphene dispersion for use as conductive ink $\mathrm{k}^{12,35,39,40}$

One of the unique properties of graphene is its molecular barrier property, which prevents gas permeation $^{3,41-43}$. We anticipated that the P3HT/graphene composite can be used for the anti-oxidation of oxidizable metal substrates in electronics. Gold- and silver-coated substrates are widely used in electronics and in electrical appliances owing to their high conductivity and high stability in the atmosphere. Although the replacement of these precious metals with base metals (e.g., $\mathrm{Cu}$ and $\mathrm{Ni}$ ) is very cost-effective, a major drawback in the electric industry is that base metals are readily oxidized in the atmosphere to produce non-conductive metal oxides. We prepared Cu-microparticles (CuMPs, $0.2 \mathrm{~mm}$ in diameter) coated with the $\mathrm{P} 3 \mathrm{HT} /$ graphene composite by dip-coating to evaluate the anti-oxidation property of the $\mathrm{P} 3 \mathrm{HT} /$ graphene coating.

Figure $5 c$ shows that the electrical resistance of bare CuMPs was $0.6 \pm 0.2 \Omega$ and that the oxidation treatment increased the resistance by more than 4 times $(2.3 \pm 0.1 \Omega)$. The P3HT-coated CuMPs exhibited a resistance of 
a)

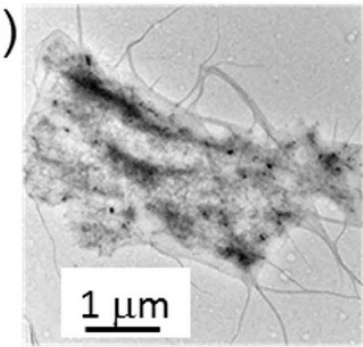

b)

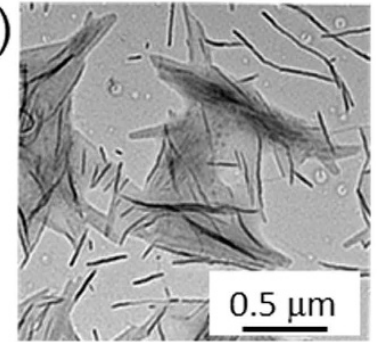

c)
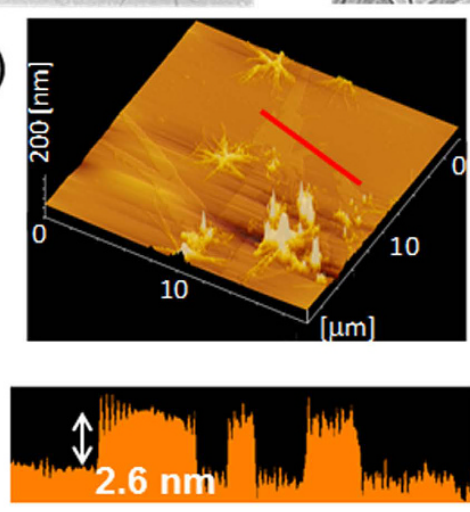

3.0

$0[\mathrm{~nm}]$

$-3.0$

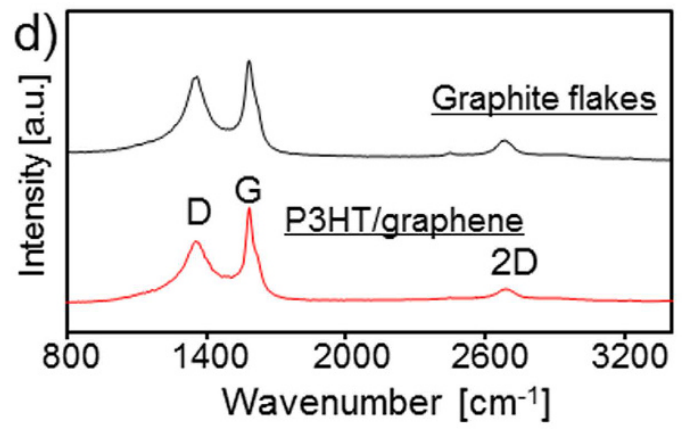

Figure 4. (a,b) TEM images of graphene sheets with P3HT $\left(M_{\mathrm{n}}=6,000\right)$. (c) AFM image of P3HT/graphene deposited on a mica substrate with a height profile along the red line. (d) Raman spectra of graphite flakes and $\mathrm{P} 3 \mathrm{HT} /$ graphene composite. P3HT $\left(M_{\mathrm{n}}=6,000\right)$ was used for preparing the P3HT/graphene complex.

$1.3 \pm 0.1 \Omega$ before oxidation and $1.6 \pm 0.3 \Omega$ after oxidation. Although the sole P3HT coating also suppressed the oxidation to a certain extent, the intrinsic resistance of the P3HT-coated CuMPs was relatively high. The P3HT/ graphene-coated CuMPs exhibited a resistance of $0.8 \pm 0.2 \Omega$ before oxidation, which was comparable with that of the bare CuMPs. After the oxidation treatment, the resistance of the P3HT/graphene-coated CuMPs remained at $0.7 \pm 0.2 \Omega$, which was as low as that of the bare CuMPs. These results show that the P3HT/graphene coating prevented $\mathrm{Cu}$ oxidation and maintained the intrinsic resistance of the CuMPs.

To verify the coatings on the CuMPs, we recorded the Raman spectra for the surfaces of the bare and coated CuMPs before and after the oxidation treatment. Figure $5 \mathrm{~d}$ shows small peaks at 600 and $220 \mathrm{~cm}^{-1}$ (red arrows in Fig. 5d) for the bare CuMPs and the P3HT-coated CuMPs after the oxidation treatment, which result from the production of $\mathrm{CuO}$ and $\mathrm{Cu}_{2} \mathrm{O}$. The observed peaks for P3HT are at 2,900 and 1,500 $\mathrm{cm}^{-1}$ (blue arrows in Fig. 5d) in the spectra of the P3HT-coated CuMPs. These results suggest successful P3HT coating of CuMPs and Cu oxidation for the P3HT-coated CuMPs by the oxidation treatment. In the spectrum of the P3HT/graphene-coated CuMPs, no peaks for copper oxide were observed while peaks for graphene (D, G, and 2D band) and peaks for $\mathrm{P} 3 \mathrm{HT}$ were observed, showing the inhibition of $\mathrm{Cu}$ oxidation and the successful P3HT/graphene coating. The inhibition of $\mathrm{Cu}$ oxidation is proposed to arise from the high gas-barrier property of graphene. It should be noted that a peak at $1600 \mathrm{~cm}^{-1}$ was assigned as a $\mathrm{G}$ peak of graphene $\mathrm{e}^{32,33}$.

Figure S4 (Supporting Information) shows the FE-SEM images of bare, and P3HT- and P3HT/graphene-coated CuMPs. A wrinkled morphology was observed on the surface of the P3HT-coated CuMPs in the magnified image (right photographs of Fig. S4b, Supporting Information). The P3HT/graphene-coated CuMPs also had a wrinkled surface, which was more obvious in the left of Fig. S4c than in Fig. S4b (Supporting Information). These wrinkled morphologies provided evidence for the coatings of $\mathrm{P} 3 \mathrm{HT}$ and the $\mathrm{P} 3 \mathrm{HT} /$ graphene on CuMPs.

\section{Discussion}

In this study, we first succeeded in the exfoliation of pristine graphite using well-defined P3HT to stably disperse graphene in toluene. In particular, regioregular P3HT with $M_{\mathrm{n}}$ of 6,000 was found to effectively exfoliate graphite 
a)

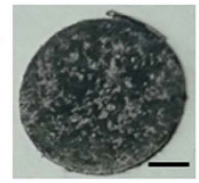

b)

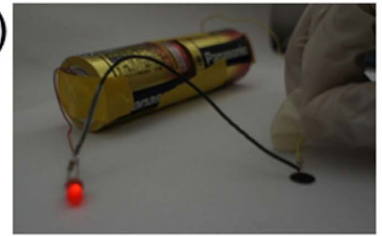

c)

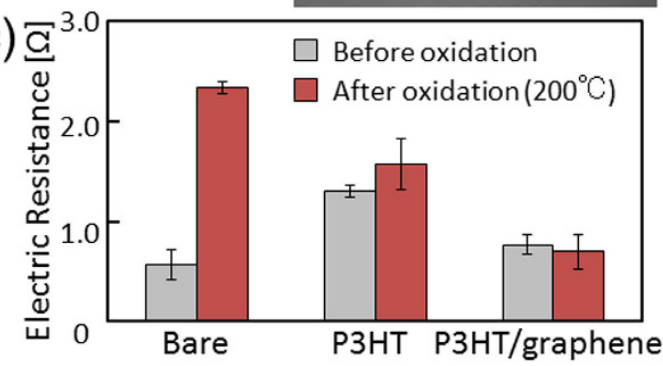

d)

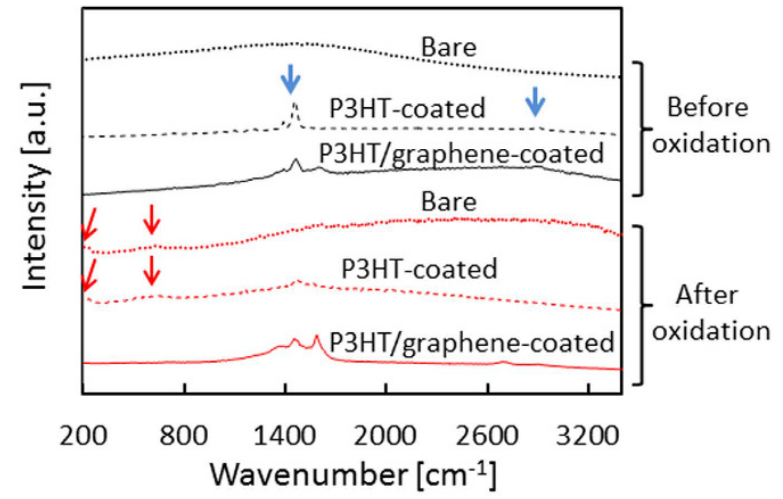

Figure 5. (a) $\mathrm{P} 3 \mathrm{HT} /$ graphene composite filtrated and collected on filter paper. Scale bar represents $2 \mathrm{~mm}$. (b) Photograph of a lit LED assembled with a P3HT/graphene composite and a battery. (c) Electrical resistance and photographs of bare CuMPs, P3HT-coated CuMPs, and P3HT/graphene-coated CuMPs before and after oxidation. (d) Raman spectra of bare CuMPs, P3HT-coated CuMPs, and P3HT/graphene-coated CuMPs before (black) and after (red) oxidation treatment.

flakes to produce graphene and to stably disperse graphene in toluene, in which $\pi-\pi$ interaction between P3HT and graphene would play an important role in the formation of the P3HT/graphene composite. The regioregularity of P3HT was also important for the exfoliation of graphite and the dispersion of graphene. The P3HT/ graphene composite showed high electrical conductivity owing to the high conductivity of P3HT and graphene, which proposes the potential application of the $\mathrm{P} 3 \mathrm{HT} /$ graphene dispersion as conductive graphene ink. The investigations on the $\mathrm{P} 3 \mathrm{HT} /$ graphene coating interestingly demonstrate that the $\mathrm{P} 3 \mathrm{HT} /$ graphene composite can be used as an oxidation-resistant and conductive film for a copper substrate. Liquid-phase exfoliation of pristine graphite and stable dispersion of graphene, without losing the properties of graphene, extend the practical applications of graphene. Although there were, to the best of our knowledge, only a few reports that proposed the application of graphene utilizing both its electrical conductivity and gas-barrier property ${ }^{36,44,45}$, the present study clearly demonstrates the high potential of the $\mathrm{P} 3 \mathrm{HT} /$ graphene composite as a gas barrier and conductive nanomaterial that is scalable and processable.

\section{References}

1. Novoselov, K. S. et al. Electric field effect in atomically thin carbon films. Science 306, 666-669 (2004).

2. Geim, A. K. \& Novoselov, K. S. The rise of graphene. Nat. Mater. 6, 183-191 (2007).

3. Novoselov, K. S. et al. A roadmap for graphene. Nature 490, 192-200 (2012).

4. Reina, A. et al. Large area, few-layer graphene films on arbitrary substrates by chemical vapor deposition. Nano Lett. 9, 30-35 (2009).

5. Kim, K. S. et al. Large-scale pattern growth of graphene films for stretchable transparent electrodes. Nature 457, 706-710 (2009).

6. Berger, C. et al. Electronic confinement and coherence in patterned epitaxial graphene. Science 312, 1191-1196 (2006).

7. Salvatierra, R. V., Cava, C. E., Roman, L. S., Oliveira, M. M. \& Zarbin, A. J. G. The total chemical synthesis of polymer/graphene nanocomposite films. Chem. Commun. 52, 1629-1632 (2016).

8. Bianco, A. et al. All in the graphene family - A recommended nomenclature for two-dimensional carbon materials. Carbon 65, 1-6 (2013).

9. Wick, P. et al. Classification framework for graphene-based materials. Angew. Chem. Int. Ed. 53, 7714-7718 (2014).

10. Laaksonen, P. et al. Interfacial engineering by proteins: exfoliation and functionalization of graphene by hydrophobins. Angew. Chem. Int. Ed. 49, 4946-4949 (2010).

11. Liang, Y. T. \& Hersam, M. C. Highly Concentrated Graphene Solutions via Polymer Enhanced Solvent Exfoliation and Iterative Solvent Exchange. J. Am. Chem. Soc. 132, 17661-17663 (2010).

12. Guardia, L. et al. High-throughput production of pristine graphene in an aqueous dispersion assisted by non-ionic surfactants. Carbon 49, 1653-1662 (2011). 
13. Wajid, A. S. et al. Polymer-stabilized graphene dispersions at high concentrations in organic solvents for composite production. Carbon 50, 526-534 (2012).

14. He, P. et al. Urea-assisted aqueous exfoliation of graphite for obtaining high-quality graphene. Chem. Commun. 51, 4651-4654 (2015).

15. Matsumoto, M., Saito, Y., Park, C., Fukushima, T. \& Aida, T. Ultrahigh-throughput exfoliation of graphite into pristine 'single-layer' graphene using microwaves and molecularly engineered ionic liquids. Nat. Chem. 7, 730-736 (2015).

16. Georgakilas, V. et al. Hydrophilic Nanotube Supported Graphene-Water Dispersible Carbon Superstructure with Excellent Conductivity. Adv. Funct. Mater. 25, 1481-1487 (2015)

17. Georgakilas, V. et al. Functionalization of graphene: covalent and non-covalent approaches, derivatives and applications. Chem Rev 112, 6156-6214 (2012).

18. McCullough, R. D. The chemistry of conducting polythiophenes. Adv. Mater. 10, 93-116 (1998).

19. Tasis, D., Tagmatarchis, N., Bianco, A. \& Prato, M. Chemistry of carbon nanotubes. Chem Rev 106, 1105-1136 (2006).

20. Kuila, B. K., Malik, S., Batabyal, S. K. \& Nandi, A. K. In-situ synthesis of soluble poly(3-hexylthiophene)/multiwalled carbon nanotube composite: Morphology, structure, and conductivity. Macromolecules 40, 278-287 (2007).

21. Chunder, A., Liu, J. H. \& Zhai, L. Reduced Graphene Oxide/Poly(3-hexylthiophene) Supramolecular Composites. Macromol. Rapid. Commun. 31, 380-384 (2010).

22. Saini, V. et al. Electrical, Optical, and Morphological Properties of P3HT-MWNT Nanocomposites Prepared by in Situ Polymerization. J. Phys. Chem. C 113, 8023-8029 (2009).

23. Yang, Z. L., Shi, X. J., Yuan, J. J., Pu, H. T. \& Liu, Y. S. Preparation of poly(3-hexylthiophene)/graphene nanocomposite via in situ reduction of modified graphite oxide sheets. Appl. Surf. Sci. 257, 138-142 (2010).

24. Bkakri, R. et al. Effects of the graphene content on the conversion efficiency of P3HT: Graphene based organic solar cells. J. Phys. Chem. Solids 85, 206-211 (2015).

25. Tamba, S. et al. Nickel-catalyzed Dehydrobrominative Polycondensation for the Practical Preparation of Regioregular Poly(3substituted thiophene)s. Chem. Lett. 40, 398-399 (2011).

26. Tamba, S., Shono, K., Sugie, A. \& Mori, A. C-H functionalization polycondensation of chlorothiophenes in the presence of nickel catalyst with stoichiometric or catalytically generated magnesium amide. J. Am. Chem. Soc. 133, 9700-9703 (2011).

27. Tanaka, S., Tamba, S., Tanaka, D., Sugie, A. \& Mori, A. Synthesis of well-defined head-to-tail-type oligothiophenes by regioselective deprotonation of 3-substituted thiophenes and nickel-catalyzed cross-coupling reaction. J. Am. Chem. Soc. 133, 16734-16737 (2011).

28. Fukumoto, H., Omori, Y. \& Yamamoto, T. Effects of solvent and temperature on regioregularity of poly(3-hexylthiophene-2,5-diyl) prepared by chemical oxidative polymerization. Polymer J. 45, 462-465 (2013).

29. Fujita, K. et al. Synthesis of Poly(3-substituted thiophene)s of Remarkably High Solubility in Hydrocarbon via Nickel-Catalyzed Deprotonative Cross-Coupling Polycondensation. Macromolecules 49, 1259-1269 (2016).

30. Gomez-Navarro, C. et al. Electronic transport properties of individual chemically reduced graphene oxide sheets. Nano Lett. 7 , 3499-3503 (2007).

31. Kudin, K. N., Ozbas, B., Schniepp, H. C., Prud'homme, R. K., Aksay, I. A. \& Car, R. Raman spectra of graphite oxide and functionalized graphene sheets. Nano Lett. 8, 36-41 (2008).

32. Ferrari, A. C. et al. Raman spectrum of graphene and graphene layers. Phys. Rev. Lett. 97 (2006).

33. Hernandez, Y. et al. High-yield production of graphene by liquid-phase exfoliation of graphite. Nat. Nanotechnol. 3, 563-568 (2008),

34. Higashi, C., Funasaki, Y., Iguchi, H. \& Maruyama, T. In-situ polymerization of a novel surfactant on graphene surface for the stable graphene dispersion in water. RSC Adv 6, 88244-88247 (2016).

35. Torrisi, F. et al. Inkjet-printed graphene electronics. ACS Nano. 6, 2992-3006 (2012).

36. Deng, B. et al. Roll-to-Roll Encapsulation of Metal Nanowires between Graphene and Plastic Substrate for High-Performance Flexible Transparent Electrodes. Nano. Lett. 15, 4206-4213 (2015).

37. Ho, D. H. et al. Stretchable and Multimodal All Graphene Electronic Skin. Adv. Mater. 28, 2601-2608 (2016).

38. http://www.sigmaaldrich.com/catalog/product/aldrich/803995.

39. Xu, Y. X., Bai, H., Lu, G. W., Li, C. \& Shi, G. Q. Flexible graphene films via the filtration of water-soluble noncovalent functionalized graphene sheets. J. Am. Chem. Soc. 130, 5856-5857 (2008).

40. Hyun, W. J. et al. All-Printed, Foldable Organic Thin-Film Transistors on Glassine Paper. Adv. Mater. 27, 7058-7064 (2015).

41. Park, S. \& Ruoff, R. S. Chemical methods for the production of graphenes. Nat. Nanotechnol. 4, 217-224 (2009).

42. Sadasivuni, K. K., Ponnamma, D., Thomas, S. \& Grohens, Y. Evolution from graphite to graphene elastomer composites. Prog. Polym. Sci. 39, 749-780 (2014).

43. Cui, Y. B., Kundalwal, S. I. \& Kumar, S. Gas barrier performance of graphene/polymer nanocomposites. Carbon 98, 313-333 (2016).

44. Chang, C. H. et al. Novel anticorrosion coatings prepared from polyaniline/graphene composites. Carbon 50, 5044-5051 (2012).

45. Lin, Y., Liu, S. Q. \& Liu, L. A new approach to construct three dimensional segregated graphene structures in rubber composites for enhanced conductive, mechanical and barrier properties. J. Mater. Chem. C 4, 2353-2358 (2016)

\section{Acknowledgements}

This work was supported partially by Special Coordination Funds for Promoting Science and Technology, Creation of Innovation Centers for Advanced Interdisciplinary Research Areas (Innovative Bioproduction Kobe), MEXT, Japan and by JSPS KAKENHI Grant Number 16H04577.

\section{Author Contributions}

All authors discussed the results and commented on the manuscript. A.N. and T.M. designed the project. H.I., K.F. and A.M. synthesized P3HT. H.I., C.H. and Y.F. performed the graphene dispersion and structural characterizations. H.I. and T.M. wrote the manuscript.

\section{Additional Information}

Supplementary information accompanies this paper at http://www.nature.com/srep

Competing financial interests: The authors declare no competing financial interests.

How to cite this article: Iguchi, H. et al. Rational and practical exfoliation of graphite using well-defined poly(3-hexylthiophene) for the preparation of conductive polymer/graphene composite. Sci. Rep. 7, 39937; doi: 10.1038/srep39937 (2017). 
Publisher's note: Springer Nature remains neutral with regard to jurisdictional claims in published maps and institutional affiliations.

(c) (i) This work is licensed under a Creative Commons Attribution 4.0 International License. The images or other third party material in this article are included in the article's Creative Commons license, unless indicated otherwise in the credit line; if the material is not included under the Creative Commons license, users will need to obtain permission from the license holder to reproduce the material. To view a copy of this license, visit http://creativecommons.org/licenses/by/4.0/

(C) The Author(s) 2017 